\title{
Research on Application of Wireless Video Surveillance System in Modern Intelligent Traffic Management
}

\author{
Jiping Ma \\ Xi'an International University, Xi'an, 710000, China
}

Keywords: Wireless video surveillance system, Intelligent traffic, Software, Application, Test.

\begin{abstract}
Along with the continuous development of science and technology, intelligent traffic wireless video surveillance system has become an important component in the traffic management system. Based on its advancement and strong ability of prevention, it can further improve the management effect. In addition, thanks to the development of embedded system and image processing technology, wireless video surveillance technology achieves a leap-forward development, able to meet the needs of social development, and conform to the new trend of video surveillance technology, so as to play its practical value. This paper briefly analyzes the software system of wireless video surveillance system in the modern intelligent traffic management, and focuses on the application program and application test, aimed to provide technical researchers more valuable technical advice.
\end{abstract}

\section{Introduction}

In the urban road management system, in order to further realize the urban road traffic comprehensive monitoring and management objectives, and further prevent illegal parking, it is necessary to actively construct effective video detection technology. The traffic violation monitoring system based on the super-resolution reconstruction technology can effectively improve the video surveillance effect, and achieve the development goal integrating detection and tracking, which can help really construct intelligent video tracking system and optimize the law enforcement efforts, and at the same time, standardize the management behavior.

\section{Software system of wireless video surveillance system in modern intelligent traffic management}

\section{Software structure based on Linux}

Based on Linux, this paper constructs the embedded system. Due to the particularity of the system hardware structure and the processor, the storage space of the whole device is not large and there is not enough space for the installation operation. Therefore, to use it as a software system, it is necessary to combined with ARM processor, to achieve cross-platform development, and establish the cross-development environment. Use the computer for program editing compilation, but also effectively download and run debugging equipment.

During the cross-environment development, all the tools in the embedded software development project needs to be integrated, mainly including text editor, cross-editor, cross-debugger and simulation structure. It should be noted that the cross-environment is composed of the host computer and the target board, and it is necessary to use physical connections to improve the effectiveness of logical connections. 1) The host computer is mainly used in computer architecture of the embedded system. The more common is the PC. It has rich hardware resources and software resources, able to provide a more complete environment for the development process. 2) In the practical application process, the target board can effectively receive and perform relevant operations to ensure that all kinds of commands can be effectively processed and controlled, mainly including setting the breakpoint structure, and reading/writing memory. It should be noted that, in the actual software system development process, with the help of physical connections, the target board and the host 
computer can be connected to enhance the logic of its connection, and optimize and coordinate related operations of the host computer.

When dealing with the embedded Linux software development, the cross-environment establishment and optimization process should be deeply integrated. First, generate the basic program, mainly proceed on the host computer, and use the cross-editing tool chain to edit and operate relevant programs to achieve the goal of connecting work, and to a certain extent, effectively generate solidifying binary target program structure. Second, effectively debug the program, use GDB to conduct concentrated debugging on completed programs, and related testing work to ensure that the overall program environment design results in line with expectations. Third, establish a sound and complete curing operation system, use the serial port, Ethernet port or USB port to solidify relevant programs, so as to effectively start the target machine, and upgrade the overall program structure based on the implementation of independent programs.

\section{Intelligent traffic wireless video terminal design}

In the software system, the application software is mainly for video capture, video data compression and video wireless transmission process. The actual embedded operating system Linux 2.6.21 ensures the effectiveness of device drivers, and uses Bootloader bootstrap program for effective analysis and integration on data process. The hardware of the whole system is S3C6410, and the main hardware interface includes USB interface (insert wireless card), UART (control signal), Camera I / F video capture chip (for analysis to achieve tracking camera, panoramic camera), MFC and so on. In the process of establishing intelligent traffic management mechanism, it is necessary to integrate terminal devices to ensure that the processing effect is more in line with actual requirements. The integrated high-speed video capture unit, video transmission unit and identification tracking unit, etc. can compress the video collected on traffic site, so as to effectively improve the optimization of wireless LAN transmission effect, and effectively detect the direction of the moving target. The motion control code sent to the camera can meet the shooting requirement for the tracking target. In the application process, the whole system structure and operation design can ensure real-time capture and transmission, improve image clarity and at the same time, provide an optimization platform for the processing performance, real-time response and reliability ${ }^{[1]}$.

\section{Application program of wireless video surveillance system in modern intelligent traffic management}

\section{Program design}

In the process of establishing and running the wireless video surveillance system, the digital technology can help image real-time monitoring of the monitored scene, and effective connection of LAN and wireless network. It should be noted that the authorized user can directly control the motion of camera PTZ lens, thus upgrading the system configuration operation results. The establishment of independent embedded system can deeply analyze the video capture process and compression process and ensure data processing and data communication optimization. First, ensure the clarity in line with standards. In the video surveillance system, only when the image clarity is ensured, the relationship between the compressed data and the transmission structure can be effectively upgraded. Second, ensure real-time performance in line with with standards. Technical staff needs the real-time management on the surveillance system, and take data compression, data decompression, data transmission and data synchronous sharing as the focus in the data integration system. Third, ensure the continuity in line with standards. Image continuity is a key item in the surveillance system. The system should integrate the embedded monitoring terminal, signal transmission network and monitoring host structure, so as to ensure the video capture effect and more effective processing measures ${ }^{[2]}$.

For example, the wireless video surveillance system is used in intelligent traffic, combined with video capture information, preprocessing process, target detection process, able to achieve concentrated location of target vehicles. As long as relevant characteristics are descripted and 
analyzed, the movement vector of the target vehicle can be initially determined and estimated. Combine with the movement state of the vehicle, and implement the control effect of PTZ, so as to carry out the target monitoring. It is important to note that in the actual measurement and processing, it is necessary to analyze the posture of the camera, to ensure that the horizontal azimuth, vertical azimuth and focal length parameters conform to the standards. Optimize the adjustment effect, and at the same time, conduct a comprehensive analysis and update on the target characteristics, so as to effectively predict the relevant information, and conduct effective capture on the abnormal behavior after entering the tracking system.

In other words, in the process of real-time video data acquisition by the embedded video surveillance terminal, it can be stored as a specific format to ensure the optimization of the effect of the buffer zone, and with the help of wireless LAN, the relevant information can be transmitted to the wireless video server host structure, to ensure the control effect in line with standards. It can also choose the embedded video surveillance terminal for the automatic analysis and tracking of mobile targets, to lay a solid foundation for the comprehensive upgrade of wireless video server host control effect. Meet the query needs of the client, and at the same time, establish WebServer system, to meet the actual needs of customers and management staff. Combined with technical projects, wireless video surveillance system work process and transmission of video signals can meet the standards. Related technical staff is required to analyze the multimedia data preprocessing technology, to avoid equipment jitter and delay during the application cache process. After analyzing the transmission protocol based on the actual needs, it can optimize the integrity of the information data processing on the basis of optimizing the actual effect of the transmission protocol, provide a guarantee for the scientific and reasonable division, and realize the sequence number demand, and at the same time clarify the timestamp of the whole structure and conduct identification processing on synchronous sources [3].

\section{Application path}

In the intelligent control mechanism for modern traffic management, we should actively implement more effective measures to really construct intelligent video surveillance platform, upgrade and transform based on the original system, and optimize its practicality. In other words, in order to further optimize the effectiveness of wireless video surveillance system, we must effectively strengthen the system design result, and implement specific requirements of auxiliary traffic management, so as to ensure that wireless video surveillance system can play its maximum value.

First, apply the wireless video surveillance system in intelligent retrieval projects. Compared to the traditional video surveillance system, wireless video surveillance system can avoid the duplicate large-scale search. The traditional video surveillance system searches effective video clips in a large number of data information clues, which not only will result in increase of the workload, and that the overall work process will also become more cumbersome. Based on this, the use of wireless video surveillance system can effectively improve the efficiency of the retrieval to ensure that the workload and work intensity meet optimization needs. After the video equipment is opened, it can effectively access the equipment operation information, centrally set different information parameters of video capture area, and fully use the kernel frame buffer area to deeply buffer and analyze on the video data. In addition, directly map it to the hard disk storage structure, to improve the retrieval effectiveness and data reliability. The whole process is finished automatically, which not only saves manpower and material resources, but also improves the practical efficiency of the retrieval process. It should be noted that, in the use of intelligent devices for retrieval and analysis, the relevant "keyword" can be input, so as to effectively extract the important content and information structure.

Second, apply the wireless video surveillance system in mobile monitoring projects. In order to improve the practical efficiency of traffic management projects, after the practical application system is established, we should actively implement more systematic control measures to strengthen the integrity of traffic management projects ${ }^{[4]}$. In other words, due to the dynamic characteristics of traffic management, the monitoring process also can meet the mobile requirements. In mobile 
monitoring, the ground traffic management personnel conducts concentrated monitoring and processing on different sections. Technical staff only needs to use handheld terminal to monitor targets in real time. After connect the wireless network, they can conduct networking operations with the video monitoring center, to establish the system for real-time transmission of the actual situation, and better grasp the traffic situation in different regions for effective implementation of relief measures to ensure the management effect and overall safety level more in line with requirements. The establishment and operation of the traffic management system should combine with the transmission function of video monitoring project for intelligent control and transmission of the traffic lights. For example, if the traffic light is flashing green, and the pedestrian is still on the crossing, the signal can turn into red with the help of the automatic control. It is this intelligent sensing and testing equipment of signal lights that helps meet the intelligent needs of traffic management when the system is networking.

\section{Application test of wireless video surveillance system in modern intelligent traffic management}

To fundamentally ensure that the wireless video surveillance system plays its actual value, it is necessary to undergo application tests. Mainly with the help of a number of test tools and test programs, test and analyze the product function and the actual performance. And design the relevant maintenance test system, to effectively analyze related issues and assessment results, and in real time track fault information so as to ensure that the developed products in line with the actual requirements, and to a certain extent, improve the effect of data processing. In the process of running the software test system, whether manual or automated testing, the required requirements and the actual results should be combined together.

The actual application test process is as follows. First, start the host computer, run the terminal system, and set the serial port transfer rate as115200bit/s, select the corresponding serial port, analyze and integrate the communication parameters, and analyze data bits, stop bit and the no parity process. Second, integrate the embedded video surveillance terminal circuit board, after power-on, load the relevant bootstrap programs, use program load operating system Linux , camera, encoding/decoding module, wireless card, etc., and so after the start of the application program, to ensure the integrity and effectiveness of testing network address information, and to a certain extent, optimize the video capture and compression effects. Third, for the intelligent traffic wireless video surveillance system, the main support format should be determined based on the actual operating environment, and currently more common formats include, CIF ,VGA and D1, able to compress and transfer with stream transmission mechanism, and ensure that the client can effectively receive and analyze video information of the central server ${ }^{[5]}$. Fourth, the client can use the personal computer to check the relevant information after logging in the program. Enter192.168.0.10, click "Enter" , the user information authentication window pops up for identification, enter the user name and password, and directly log in the main configuration page. Fifth, with a personal computer the client can open the VLC player, and with the configuration description module, read the file and display traffic conditions.

\section{Conclusions}

All in all, based on the continuous development and progress of information technology in China, the application of wireless video surveillance system in intelligent traffic project has become the inevitable trend of social development. And the relevant departments need to establish and improve more systematic control measures and management mechanism, link together technical structure and actual needs and upgrade design structure and development effects, to provide protection for promotion of upgrading technology applications, construct network operations of surveillance system, and achieve the intelligent development, as well the comprehensive upgrade of the intelligent level of our traffic management. 


\section{References}

[1] Liu Cheng, Duan Hongguang, Ba Yi, et al. Research and Design of Mobile Video Surveillance System Client Based on Android, Television Technology, 2013, 37 (04): 85-88.

[2] Cao Yi. Application of Wireless Video Surveillance Technology in On-board Video Surveillance System, Beijing University of Posts and Telecommunications, 2013.

[3] He Dong. Perfect Solutions of Wireless Intelligent Traffic and Video Surveillance of US Henrich Electronics, 2015 (Sixth) Industrial Ethernet Technology and Application Forum Proceedings. 2015: 117-131.

[4] Li Qiulin. Design and Implementation of Wireless Vehicle Video Monitor Terminal Based on OMAP, Guangdong University of Technology, 2015.

[5] Li Yanjun. Research and Implementation of New 3G Vehicle Mobile Terminal in Intelligent Traffic Guidance System, Nanjing University of Posts and Telecommunications, 2016. 